\section{The grade of neoangiogenesis and the bone marrow blast count predict leukemic transformation-free survival in myelodysplastic syndromes}

\section{DOI: $10.1515 / \mathrm{rrlm}-2016-0020$}

Myelodysplastic syndromes (MDS) are clonal bone marrow disorders showing highly variable clinical courses. The median overall survival in MDS is 15-30 months, the risk of acute myeloid leukemia (AML) transformation being 25-30\% at five years (1). It is known, that blasts' percentage, karyotypic abnormalities and the depth of cytopenias included in the International Prognostic Scoring System (IPSS) are strong predictors for a poor prognosis and AML transformation risk in MDS (2). In a previous regional study, Bănescu et al. revealed a $62.5 \%$ incidence of abnormal karyotypes in MDS patients. According to their findings, the leukemic transformation-risk was higher and the overall survival shorter in cases with cytogenetic alterations and especially, with complex karyotype (3). The IPSS does not have an absolute value since half of the MDS cases appear with normal karyotype (4). In parallel, there is an increasing endeavour to focus research on the MDS-AML interface, crucial in the disease progression. The molecular mechanism of leukemic transformation in MDS is still incompletely understood, but most of the recently detected molecular changes are present only in a fraction of MDS patients (5), (6). For these considerations, no universal molecular model can be applied, multi-step pathways of leukemogenesis being influenced by the balance of apoptosis and proliferation, host-response related features and stromal reactions (7). In MDS, there is a growing interest for histopathological diagnosis based on the percentage, morphological and immunophenotypic features of blasts in the bone marrow (according to the WHO 2008 classification). On the other hand, the histological subtypes have been included in risk models of MDS-AML transformation. The trephine biopsy can offer important clues for diagnosis since it characterizes the complex interaction between the stroma as microenvironment, the normal and dysplastic cells.

The angiogenic factors produced by the neoplastic and non-neoplastic cell types play a role in angiogenesis, microvessel density being described as an independent prognostic parameter in solid tumours. An aberrant expression of vascular endothelial growth factor (VEGF) has also been revealed in atypical localization of immature precursors (ALIPs) of BM biopsies taken from MDS patients, suggesting that VEGF levels might play a role not only in neovascularisation, but also in the delivery of autocrine and paracrine stimuli for blasts, thus having a role in the mechanism of transformation (7). The number of studies is limited, but the results of these studies focused on microvessel density (MVD) confirm that neither MVD nor VEGF expression were predictors of progression to AML, although they confirmed increased MVD in MDS (8), (9), (10).

We studied angiogenesis in 47 cases of myelodysplastic syndrome by a quantitative morphometric approach. An informed consent was obtained from each patient and the Ethics Committee of the Emergency Clinical Hospital issued the approval no. 780/14.01.2016 for the study. Microvessel density was evaluated on archival CD 34 immunostained bone marrow trephine biopsy specimens. CD34 staining of blast cells was eliminated by oversizing the capture threshold in 
comparison to the blast diameter. For each case, ten microscopic fields were chosen, captured with a 20x objective, saved in JPEG format and imported for analysis into the Image $\mathrm{J}$ software for determining the endothelial surface area (Image processing and analysis in Java, National Institutes of Health). The final result was calculated individually for each case based on the arithmetic mean. Morphometric results were correlated with clinical data, with the blast count, and low- and high- risk groups were defined. The Kaplan-Meier analysis was conducted to describe the leukemic transformation-free period.

In the studied cohort, cases were classified according to the WHO criteria, as refractory anaemia (RA), refractory anaemia with blast excess (RAEB-1, RAEB-2) and refractory cytopenia with multi-lineage dysplasia (RCMD). The overall observation period was 42 months, the survival was 10 (4-24) months and 9 patients (19\%) developed acute myeloid leukaemia.

We observed significant differences between the CD34 expression levels of patient subgroups with leukemic transformation and with stable disease -2.69 (1.22-4.24) vs. 0.85 (0.47-1.92), and those who show immediate aggravation with a fatal non-leukemic outcome- 0.70 (0.20-0.87) - $\mathrm{p}=0.008, \mathrm{r}=0.44$ and $\mathrm{p}=0.012, \mathrm{r}=0.59$, respectively. Taking these into account, we applied two types of time-to-transformation risk classification criteria to the cohort:

3. Simple risk classification based on the bone marrow blast count rank (low-risk: $<10 \%$, high-risk $>10 \%$, (30 vs. 17 cases).

4. Combined risk classification based on a score obtained from the blast count and the angiogenic CD34 expression level. We applied many models including the two parameters and finally applied the one based on the following formula:

\begin{tabular}{|c|c|c|c|}
\hline $\begin{array}{c}\text { CR } \\
\text { score }\end{array}$ & $=\begin{array}{c}(\text { Blast count } \\
\text { rank })\end{array}$ & $\mathrm{x}$ & $\begin{array}{c}\text { (CD34 expression } \\
\text { rank) }\end{array}$ \\
\hline
\end{tabular}

where "blast count rank" was interpreted similarly $(1-<10 \%, 2->10 \%)$, the CD34 expression rank was ordered according to the tertiles of the values obtained on the cohort -1, 2, 3. CR scores 1, 2, 3 were considered at low risk while 4 and 6 at high-transformation risk ( 38 vs. 9 cases).

According to the simple risk classification, $11.5 \%$ from the low-risk group, and $35.3 \%$ of cases from the high-risk group suffered a leukemic transformation $(p=0.12)$. At the combined risk classes, we observed that $9.4 \%$ of the lowrisk versus $54.5 \%$ of the high-risk individuals developed AML $(p=0.016)$.

In the case of simple risk groups, Kaplan-Meier analysis for the transformation-free survival revealed that the curves were significantly different $(\log$-rank test $\mathrm{p}=0.021$, Cox-Mantel test $\mathrm{p}=0.005)$. At the combined risk classification, the difference was more relevant $(\log$ rank test $\mathrm{p}=0.007$, Cox-Mantel test $\mathrm{p}<0.001$ ) (Figure 1).

Thus, both risk classifications determined valid risk groups, the combined classification offering a stronger transformation-risk prediction.

International initiatives tend to improve and refine the IPSS variables, suggesting inclusion of the serum lactate dehydrogenase activity, ferritin and beta- 2 microglobulin as well as bone marrow fibrosis. Our data show that bone marrow angiogenesis could be another factor to consider. In order to establish the exact role of bone marrow angiogenesis in the MDS-AML interface, larger studies on multiethnic cohorts should be performed.

\section{Conflict of interest}

The authors hereby declare no conflict of interest.

\section{Role of funding source}

This work was partly supported by the research contract no. 6/30.10.2013 with the Studium-Prospero Foundation. 


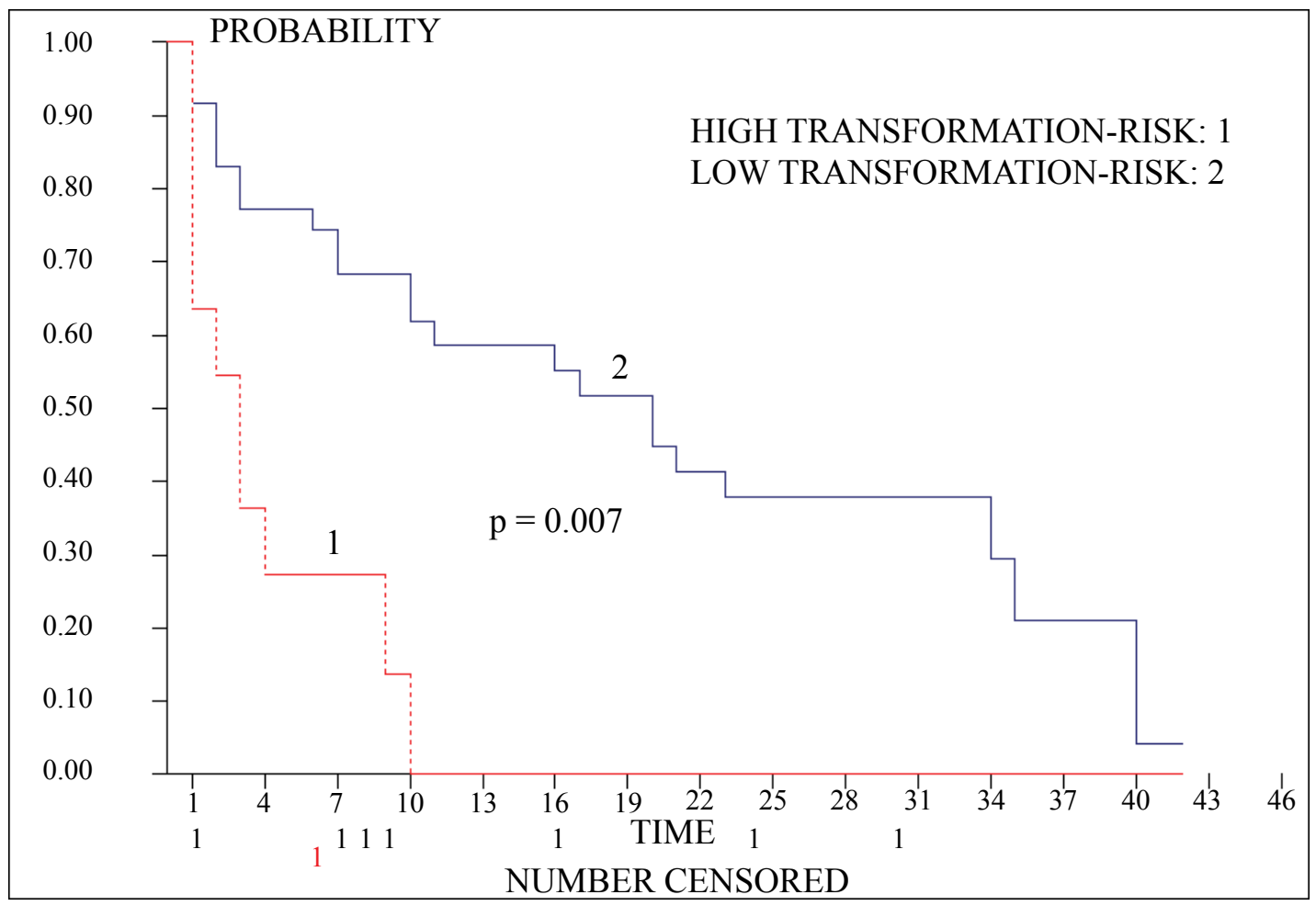

Figure 1. Kaplan-Meier curves for transformation-free survival in the combined risk group 1 and 2. P value for log-rank test is shown

\section{Horváth Emőke ${ }^{1}$, Finna Csilla ${ }^{1}$, Demian Smaranda ${ }^{2}$, Nagy Előd-Erno" ${ }^{3 *}$}

1. Department of Pathology, University of Medicine and Pharmacy, Târgu-Mures, Romania.

2. University of Medicine and Pharmacy, Hematology Clinic 1, Emergency Clinical Hospital, Târgu-Mures, Romania.

3. Department of Pharmaceutical Biochemistry, University of Medicine and Pharmacy, Târgu-Mures, Romania.

*Corresponding author: Nagy Elöd-Ernő, elod.nagy@umftgm.ro

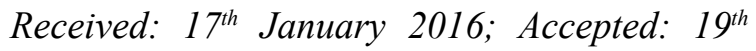
April 2016; Published: 31 $1^{\text {th }}$ May 2016.

\section{References}

1. Fenaux P, Haase D, Sanz GF, Santini V, Buske C. Clinical practice guidelines Myelodysplastic syndromes: ESMO Clinical Practice Guidelines for diagnosis, treatment and follow-up. Ann Oncol. 2014 Sep;25(Suppl 3):57-69. DOI: 10.1093/annonc/mdu180.

2. Greenberg PL, TuechlerH, SchanzJ, SanzG, Garcia-Manero G, Solé F, et al. Revised International Prognostic Scoring System for Myelodisplastic Syndromes. Blood. 2012 Sep; 120(12):2454-65. DOI: 10.1182/blood-2012-03-420489.

3. Bănescu C, Benedek I, Duicu C, Demian S, Voidăzan $\mathrm{S}$. Cytogenetic findings and their prognostic impact in myelodysplastic syndrome patientes. Revista Română de Medicină de Laborator. 2011 Iun;2(19):139-47.

4. Duarte FB, Goncalves RP, Barbosa MC, Rocha Filho FD, de Jesus dos Santos TE, Nogueira dos Santos T, et al. Tumor suppressor p53 protein expression: prognostic significance in patients with low-risk myelodysplastic syndrome. Rev Bras Hemathol Hemother. 2014 Apr;36(3):196-201. DOI: 10.1016/j.bjhh.2014.03.007. 
5. Nolte F, Hofmann WK. Molecular mechanisms involved in the progression of myelodysplastic syndrome. Future Oncol. 2010 Mar;6(3):445-55. DOI: 10.2217/fon.09.175.

6. Bejar RK, Stevenson K, Abdel-Wahab O, Galili N, Nilsson B, Garcia-Manero G, et al. Clinical effect of point mutation in Myelodysplastic Syndromes. N Engl J Med. 2011 Iun; 364: 2496-506.

7. Porwit A, Saft L. The AML-MDS interface-leukemic transformation in myelodysplastic syndromes. J Hematop. 2011 May;4(2):69-79. DOI: 10.1007/ s12308-011-0088-6.

8. Savic A, Cemerikic-Martinovic V, Dovat S, Rajic N, Urosevic I, Sekulic B, et al. "Angiogenesis and survival in patients with myelodysplastic syndrome". Pathol Oncol Res. 2012 Jul;18(3):681-90. DOI: 10.1007/ s12253-012-9495-y.
9. Cheng CL, Hou HA, Jhuang JY, Lin CW, Chen CY, Tang JL, et al. High bone marrow angiopoietin-1 expression is an independent poor prognostic factor for survival in patients with myelodysplastic syndromes. Br J Cancer. 2011 Aug;105(7);975-82. DOI: 10.1038/ bjc.2011.340.

10. Ribatti D, Polimeno G, Vacca G, Marzullo A, Crivellato E, Nico B, et al. Correlation of bone marrow angiogenesis and mast cells with tryptase activity in myelodysplastic syndromes. Leukemia. 2002 Sep;16(9):1680-4. DOI: $10.1038 /$ sj.leu.2402586. 